Review

\title{
An Overview of the Pathway Idea and Its Applications in Statistical and Physical Sciences
}

\author{
Nicy Sebastian ${ }^{1, *}$, Seema S. Nair ${ }^{2}$ and Dhannya P. Joseph ${ }^{3}$ \\ ${ }^{1}$ St.Thomas' College, Thrissur P.O., Kerala 680001, India \\ ${ }^{2}$ St. Gregorios College, Pulamon P.O., Kottarakara, Kerala 691531, India; \\ E-Mail: seema.cms@gmail.com \\ ${ }^{3}$ Kuriakose Elias College, Mannanam P.O., Kottayam, Kerala 686561, India; \\ E-Mail: dhannyapj@gmail.com \\ * Author to whom correspondence should be addressed; E-Mail: nicyseb@yahoo.com; \\ Tel.: +91-949-525-4050.
}

Academic Editor: Hans J. Haubold

Received: 18 October 2015 / Accepted: 7 December 2015 / Published: 19 December 2015

\begin{abstract}
Pathway idea is a switching mechanism by which one can go from one functional form to another, and to yet another. It is shown that through a parameter $\alpha$, called the pathway parameter, one can connect generalized type- 1 beta family of densities, generalized type- 2 beta family of densities, and generalized gamma family of densities, in the scalar as well as the matrix cases, also in the real and complex domains. It is shown that when the model is applied to physical situations then the current hot topics of Tsallis statistics and superstatistics in statistical mechanics become special cases of the pathway model, and the model is capable of capturing many stable situations as well as the unstable or chaotic neighborhoods of the stable situations and transitional stages. The pathway model is shown to be connected to generalized information measures or entropies, power law, likelihood ratio criterion or $\lambda$-criterion in multivariate statistical analysis, generalized Dirichlet densities, fractional calculus, Mittag-Leffler stochastic process, Krätzel integral in applied analysis, and many other topics in different disciplines. The pathway model enables one to extend the current results on quadratic and bilinear forms, when the samples come from Gaussian populations, to wider classes of populations.
\end{abstract}


Keywords: pathway model; entropy measure; superstatistics; Tsallis statistics; beta family; generalized gamma; Dirichlet densities; $\lambda$-criterion; $H$-function; quadratic forms

MSC classifications: $85 \mathrm{~A} 99 ; 82 \mathrm{~B} 31 ; 60 \mathrm{E} 05 ; 62 \mathrm{C} 10 ; 33 \mathrm{C} 60 ; 44 \mathrm{~A} 15$

\section{Introduction}

The pathway idea was originally prepared by Mathai in the 1970's in connection with population models, and later rephrased and extended, Mathai [1], to cover scalar as well as matrix cases as made suitable for modelling data from statistical and physical situations. For practical purposes of analyzing data of physical experiments and in building up models in statistics, we frequently select a member from a parametric family of distributions. But it is often found that the model requires a distribution with a thicker or thinner tail than the ones available from the parametric family, or a situation of right tail cut-off. The experimental data reveal that the underlying distribution is in between two parametric families of distributions. In order to create a pathway from one functional form to another, a pathway parameter is introduced and a pathway model is created in Mathai [1]. The main idea behind the derivation of this model is the switching properties of going from one family of functions to another and yet another family of functions. The model enables one to proceed from a generalized type- 1 beta model to a generalized type- 2 beta model to a generalized gamma model when the variable is restricted to be positive. Thus the pathway parameter $\alpha$ takes one to three different functional forms. This is the distributional pathway. More families are available when the variable is allowed to vary over the real line. Mathai [1] deals mainly with rectangular matrix-variate distributions and the scalar case is a particular case there. For the real scalar case the pathway model is the following:

$$
f_{1}(x)=c_{1} x^{\gamma-1}\left[1-a(1-\alpha) x^{\delta}\right]^{\frac{\eta}{1-\alpha}}
$$

$a>0, \delta>0,1-a(1-\alpha) x^{\delta}>0, \gamma>0, \eta>0$ where $c_{1}=\frac{\delta(a(1-\alpha))^{\frac{\gamma}{\delta}} \Gamma\left(\frac{\eta}{1-\alpha}+1+\frac{\gamma}{\delta}\right)}{\Gamma\left(\frac{\gamma}{\delta}\right) \Gamma\left(\frac{\eta}{1-\alpha}+1\right)}$ for $\alpha<1$, is the normalizing constant if a statistical density is needed and $\alpha$ is the pathway parameter. For $\alpha<1$ the model remains as a generalized type- 1 beta model in the real case. Other cases available are the regular type-1 beta density, Pareto density, power function, triangular and related models. Observe that Equation (1) is a model with the right tail cut off. When $\alpha>1$ we may write $1-\alpha=-(\alpha-1), \alpha>1$ so that $f(x)$ assumes the form,

$$
f_{2}(x)=c_{2} x^{\gamma-1}\left[1+a(\alpha-1) x^{\delta}\right]^{-\frac{\eta}{\alpha-1}}, x>0
$$

which is a generalized type-2 beta model for real $x$ and $c_{2}=\frac{\delta(a(\alpha-1))^{\frac{\gamma}{\delta}} \Gamma\left(\frac{\eta}{\alpha-1}\right)}{\Gamma\left(\frac{\gamma}{\delta}\right) \Gamma\left(\frac{\eta}{\alpha-1}-\frac{\gamma}{\delta}\right)}$ for $\alpha>1$, is the normalizing constant, if a statistical density is required. Beck and Cohen's superstatistics belong to this case Equation (2) (for more details see, [2,3]). Again, dozens of published papers are available on the topic of superstatistics in statistical mechanics. For $\gamma=1, a=1, \delta=1$ we have Tsallis statistics for $\alpha>1$ from Equation (2) (for more details [4,5]). Other standard distributions coming from this model 
are the regular type-2 beta, the F-distribution, Lévi models and related models. When $\alpha \rightarrow 1$, the forms in Equations (1) and (2) reduce to

$$
f(x)=c x^{\gamma-1} \mathrm{e}^{-\mathrm{bx} \delta}, x>0, b=a \eta
$$

where $c=\frac{\delta b}{\Gamma\left(\frac{\gamma}{\delta}\right)}$, is the normalizing constant. This includes generalized gamma, gamma, exponential, chisquare, Weibull, Maxwell-Boltzmann, Rayleigh, and related models (for more details see, [6,7]). If $x$ is replaced by $|x|$ in Equation (1) then more families of distributions are covered in Equation (1). The behavior of the pathway model for various values of the pathway parameter $\alpha$ can be seen from the following figures.

From the Figure 1(Left) we can see that, as $\alpha$ moves away from 1 the function $f_{2}(x)$ moves away from the origin and it becomes thicker tailed and less peaked. From the path created by $\alpha$ we note that we obtain densities with thicker or thinner tail compared to generalized gamma density. From Figure 1 (Right) we can see that when $\alpha$ moves from $-\infty$ to 1 , the curve becomes thicker tailed and less peaked, see $([8,9])$. Note that $\alpha$ is the most important parameter here for enabling one to more than one family of functions. The other parameters are the usual parameters within each family of functions. The following is a list of some particular cases and the transformations are listed to go from the extended versions to the regular cases.
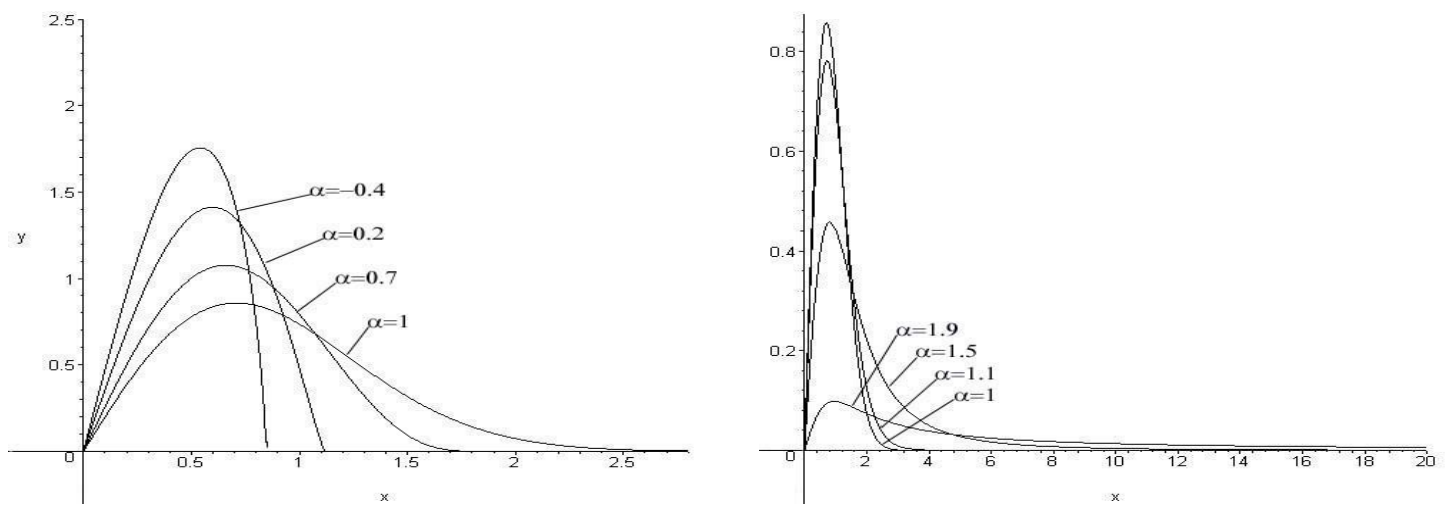

Figure 1. (Left) The graph of $f_{1}(x)$, for $\gamma=\eta=a=1, \delta=2$ and for various values of $\alpha$. (Right) The graph of $f_{2}(x)$, for $\gamma=\eta=a=1, \delta=2$ and for various values of $\alpha$.

\begin{tabular}{|c|c|}
\hline$\alpha=1, \gamma=1, a=1, \delta=1$ & Gaussian or normal density for $\infty<x<\infty$ \\
\hline$\alpha=1, \gamma-1=\frac{3}{4}, a=1, \delta=1$ & Maxwell-Boltzmann density \\
\hline$\alpha=1, \gamma-1=\frac{1}{2}, a=1, \delta=1$ & Rayleigh density \\
\hline$\alpha=1, \gamma=\frac{n}{2}, a=1, \delta=1$ & Hermert density \\
\hline$\alpha=0, \gamma=1, \eta=1, \delta=1$ & U-shaped density \\
\hline$\alpha=2, \gamma=1, \eta=\frac{\nu+1}{2} a=\frac{1}{\nu}, \delta=1$ & Student-t for $\nu$ degrees of freedom, $-\infty<x<\infty$ \\
\hline$\alpha=2, \eta=1, a=1, \delta=1$ & Caushy density for $-\infty<x<\infty$ \\
\hline$\alpha<1, a(1-\alpha)=1, \delta=1$ & Standard type-1 beta density \\
\hline
\end{tabular}




\begin{tabular}{|c|c|}
\hline$\alpha>1, a(1-\alpha)=1, \delta=1$ & Standard type- 2 beta density \\
\hline$\gamma-1=\frac{1}{2}, \eta=1, a=1, \delta=1$ & $\begin{array}{l}\text { Tsallis statistics in Astrophysics, } \\
\text { Power law, } q \text {-binomial density }\end{array}$ \\
\hline$\alpha=0, \gamma-1=\frac{1}{2}, \eta=1, \delta=1$ & Triangular density \\
\hline$\alpha=2, \gamma-\frac{1}{2}=\frac{m}{2}, a=\frac{m}{n} \eta=\frac{m+1}{2}, \delta=1$ & F-density \\
\hline$\alpha=1, \gamma-1=\frac{1}{2}, a=1, \eta=\frac{m g}{K T}, \delta=1$ & Helley's density in physics \\
\hline$\alpha=1, a=1, \delta=1$ & Gamma density \\
\hline$\alpha=1, a=1, \gamma-\frac{1}{2}=\frac{\nu}{2}, \eta=\frac{1}{2}, \delta=1$ & Chisquare density for $\nu$ degrees of freedom \\
\hline$\alpha=1, a=1 \gamma-1=\frac{1}{2}, \delta=1$ & $\begin{array}{l}\text { Exponential density (Laplace density } \\
\quad \text { with } x=|z|, \quad-\infty<z<\infty \text { ) }\end{array}$ \\
\hline$\alpha=1, a=1$ & Generalized gamma density \\
\hline$\alpha=1, a=1, \gamma-1=\frac{1}{2}$ & Weibull density \\
\hline$\alpha=2, a=1, \gamma-1=\frac{1}{2}, \eta=2, \delta=1, x=\mathrm{e}^{y}$ & Logistic density for $-\infty<y<\infty$ \\
\hline$\alpha=2, a=1, \gamma=1, \eta=1, \delta=1, x=\mathrm{e}^{\epsilon+\mu y}, \epsilon \neq 0, \mu>0$ & Fermi-Dirac density, $0 \leq y<\infty$ \\
\hline
\end{tabular}

\subsection{Pathway Model from Mathai's Entropy Measure}

In physical situations when an appropriate density is selected, one procedure is the maximization of entropy. Mathai and Rathie [10] consider various generalizations of Shannon entropy measure and describe various properties including additivity, characterization theorem etc. Mathai and Haubold ([11]) introduced a new generalized entropy measure which is a generalization of the Shannon entropy measure. For a multinomial population $P=\left(p_{1}, \ldots, p_{k}\right), p_{i}>0, i=1, \ldots, k, p_{1}+p_{2}+\cdots+p_{k}=1$, the Mathai's entropy measure is given by the relation

$$
\begin{gathered}
\sum_{k, \alpha}(P)=\frac{\sum_{i=1}^{k} p_{i}^{2-\alpha}-1}{\alpha-1}, \alpha \neq 1,-\infty<\alpha<2 . \quad \text { (discrete case) } \\
M_{\alpha}(f)=\frac{1}{\alpha-1}\left[\int_{-\infty}^{\infty}[f(x)]^{2-\alpha} \mathrm{d} x-1\right], \alpha \neq 1, \alpha<2 \quad \text { (continuous case) }
\end{gathered}
$$

By optimizing Mathai's entropy measure, one can arrive at pathway model of Mathai [1], which consists of many of the standard distributions in statistical literature as special cases. For fixed $\alpha$, consider the optimization of $M_{\alpha}(f)$, which implies optimization of $\int_{x}[f(x)]^{2-\alpha} \mathrm{d} x$, subject to the following conditions:

(i) $f(x) \geq 0$, for all $x$

(i) $\int_{x} f(x) \mathrm{d} x<\infty$

(i) $\int_{x} x^{\rho(1-\alpha)} f(x) \mathrm{d} x=$ fixed for all $f$ 
(i) $\int_{x} x^{\rho(1-\alpha)+\delta} f(x) \mathrm{d} x=$ fixed for all $f$, where $\rho$ and $\delta$ are fixed parameters

By using calculus of variation, one can obtain the Euler equation as

$$
\begin{aligned}
& \frac{\partial}{\partial f}\left[f^{2-\alpha}-\lambda_{1} x^{\rho(1-\alpha)} f+\lambda_{2} x^{\rho(1-\alpha)+\delta} f\right]=0 \\
& \Rightarrow(2-\alpha) f^{1-\alpha}=\lambda_{1} x^{\rho(1-\alpha)}\left[1-\frac{\lambda_{2}}{\lambda_{1}} x^{\delta}\right], \alpha \neq 1,2 \\
& \Rightarrow f_{1}=c_{1} x^{\rho}\left[1-a(1-\alpha) x^{\delta}\right]^{\frac{1}{1-\alpha}}
\end{aligned}
$$

for $\frac{\lambda_{2}}{\lambda_{1}}=a(1-\alpha)$ for some $a>0$. For more details see ([12,13]).

When $\alpha \rightarrow 1$, the Mathai's entropy measure $M_{\alpha}(f)$ goes to the Shannon entropy measure and this is a variant of Havrda-Charvát entropy, and the variant form therein is Tsallis entropy. Then when $\alpha$ increases from $1, M_{\alpha}(f)$ moves away from Shannon entropy. Thus $\alpha$ creates a pathway moving from one function to another, through the generalized entropy also. This is the entropic pathway. One can derive Tsallis statistics and superstatistics (for more details see, [2-5]) by using Mathai's entropy.

The pathway parameter $\alpha$ offers the differential pathway also. Let us consider

$$
\begin{aligned}
g(x) & =\frac{f_{1}(x)}{c_{1}}=x^{\beta}\left[1-a(1-\alpha) x^{\delta}\right]^{\frac{1}{1-\alpha}}, x>0, \beta=\gamma-1, \eta=1 \\
\frac{\mathrm{d}}{\mathrm{d} x} g(x) & =\frac{\beta}{x} g(x)-a \delta x^{\delta-1+(1-\alpha) \gamma}[g(x)]^{\alpha} \\
& =-a[g(x)]^{\alpha} \text { for } \beta=0, \delta=1
\end{aligned}
$$

This is the power law. When $\eta=1$ then the differential equation satisfied by $g_{3}=\frac{f}{c}$ of Equation (3) is given by

$$
\begin{aligned}
\frac{\mathrm{d}}{\mathrm{d} x} g_{3}(x) & =\frac{\beta}{x}-a \delta x^{\delta-1} g_{3}(x) \\
& =-a\left[g_{3}(x)\right] \text { for } \beta=\gamma-1=0, \delta=1
\end{aligned}
$$

Thus when $\alpha$ moves to 1 the differential pathway is from the power law in Equation (5) to the maxwell-Boltzmann in Equation (6).

\subsection{Laplacian Density and Stochastic Processes}

The real scalar case of the pathway model in Equation (1), when $x$ is replaced by $|x|$ and $\alpha \rightarrow 1$, takes the form

$$
f_{3}(x)=c_{3}|x|^{\gamma-1} \mathrm{e}^{-a|x|^{\delta}},-\infty<x<\infty, a>0
$$

The density in Equation (7) for $\gamma=1, \delta=1$ is the simple Laplace density. For $\gamma=1$ we have the symmetric Laplace density. A general Laplace density is associated with the concept of Laplacianness of quadratic and bilinear forms. For the concept of Laplacianness of bilinear forms, corresponding to the chisquaredness of quadratic forms, and for other details see [14,15]. Laplace density is also connected to input-output type models. Such models can describe many of the phenomena in nature. When two particles react with each other and energy is produced, part of it may be consumed or converted or lost and what is usually measured is the residual effect. The water storage in a dam is the residual effect of 
the water flowing into the dam minus the amount taken out of the dam. Grain storage in a sylo is the input minus the grain taken out. It is shown in Mathai ([16]) that when we have gamma type input and gamma type output the residual part $z=x-y, x=$ input variable, $y=$ output variable, then the special cases of the density of $z$ is a Laplace density. In this case one can also obtain the asymmetric Laplace and generalized Laplace densities, which are currently used very frequently in stochastic processes, as special cases of the input-output model. Some aspects of the matrix version of the input-output model is also described in [16].

\subsection{Mittag-Leffler Density and Processes}

Recently there is renewed interest in Mittag-Leffler function as a model in many applied areas due to many reasons, one being that it gives a thicker tail compared to the exponential model. Fractional differential equations often lead to Mittag-Leffler functions and their generalizations as solutions, especially when dealing with fractional equations in reaction-diffusion problems. A large number of such situations are illustrated in $[13,17,18]$. The Mittag-Leffler density, associated with a 3-parameter Mittag-Leffler function is the following:

$$
f(x)=\frac{x^{\alpha \beta-1}}{\delta^{\beta}} \sum_{k=0}^{\infty} \frac{(\beta)_{k}}{k !} \frac{\left(-x^{\alpha}\right)^{k}}{\delta^{k} \Gamma(\alpha k+\alpha \beta)}, 0 \leq x<\infty, \delta>0, \beta>0
$$

It has the Laplace transform

$$
L_{f}(t)=\left[1+\delta t^{\alpha}\right]^{-\beta}, 1+\delta t^{\alpha}>0
$$

If $\delta$ is replaced by $\delta(q-1)$ and $\beta$ by $\frac{\beta}{(q-1)}, q>1$ and if we consider $q$ approaching to 1 then we have

$$
\lim _{q \rightarrow 1} L_{f}(t)=\lim _{q \rightarrow 1}\left[1+\delta(q-1) t^{\alpha}\right]^{-\frac{\beta}{q-1}}=\mathrm{e}^{-\delta \beta t^{\alpha}}
$$

But this is the Laplace transform of a constant multiple of a positive Lévy variable with parameter $\alpha, 0<\alpha \leq 1$, with the multiplicative constant being $(\delta \beta)^{\frac{1}{\alpha}}$, and thus the limiting form of a Mittag-Leffler distribution is a Lévy distribution. A connection of pathway model to Mittag-Leffler function is given in $[19,20]$. There is vast literature on Mittag-Leffler stochastic processes, see for example [21,22].

\subsection{Laplace Transform of the Pathway Model}

Let $L_{f_{2}}(t)$ be the Laplace transform of the pathway model $f_{2}(x)$ of Euqation (2). That is

$$
\begin{gathered}
L_{f_{2}}(t)=c_{2} \int_{0}^{\infty} \mathrm{e}^{-t x} x^{\gamma-1}\left[1+a(\alpha-1) x^{\delta}\right]^{-\frac{\eta}{\alpha-1}} \mathrm{~d} x, \\
a>0, b>0, \delta>0, \\
\eta>0, \alpha>1
\end{gathered}
$$

Here the integrand can be taken as a product of positive integrable functions and then we can apply Mellin transform and inverse Mellin transform technique to evaluate the above integral. The integral in Equation (11) can be looked upon as the Mellin convolution of exponential density and superstatistics. Let us transform $x_{1}$ and $x_{2}$ to $u=\frac{x_{1}}{x_{2}}$ and $v=x_{2}$, then we can see that the marginal density of $u$ obtained 
is actually the Laplace transform that we want to evaluate. Since the density is unique, in whatever way we evaluate the density we should get the same function or the functions will be identical. We can evaluate the density of $u$ by the method of inverse Mellin transform, see [9]. Comparing the density obtained in these two different methods, we will get the Laplace transform of the pathway model given in Equation (2) as an $H$-function

$$
L_{f_{2}}(t)=\frac{1}{\Gamma\left(\frac{\gamma}{\delta}\right) \Gamma\left(\frac{\eta}{\alpha-1}-\frac{\gamma}{\delta}\right)} H_{1,2}^{2,1}\left[\left.\frac{t}{a^{\frac{1}{\delta}}(\alpha-1)^{\frac{1}{\delta}}}\right|_{(0,1),\left(\frac{\eta}{\alpha-1}-\frac{\gamma}{\delta}-\frac{1}{\delta}, \frac{1}{\delta}\right)} ^{\left(1-\frac{\gamma}{\delta} \frac{1}{\delta}\right)}\right],
$$

for $\Re(\gamma)>0, \Re\left(\frac{\eta}{\alpha-1}-\frac{\gamma}{\delta}\right)>0, \alpha>1$, where $H$ - function is defined as

$$
H_{p, q}^{m, n}\left[\left.z\right|_{\left(b_{1}, \beta_{1}\right), \ldots, \ldots,\left(b_{q}, \beta_{q}\right)} ^{\left(a_{1}, \alpha_{1}\right), \ldots,\left(a_{p}, \alpha_{p}\right)}\right]=\frac{1}{2 \pi i} \int_{L} \phi(s) z^{-s} \mathrm{~d} s
$$

where

$$
\phi(s)=\frac{\left\{\prod_{j=1}^{m} \Gamma\left(b_{j}+\beta_{j} s\right)\right\}\left\{\prod_{j=1}^{n} \Gamma\left(1-a_{j}-\alpha_{j} s\right)\right\}}{\left\{\prod_{j=m+1}^{q} \Gamma\left(1-b_{j}-\beta_{j} s\right)\right\}\left\{\prod_{j=n+1}^{p} \Gamma\left(a_{j}+\alpha_{j} s\right)\right\}}
$$

where $\alpha_{j}, j=1,2, \ldots, p$ and $\beta_{j}, j=1,2, \ldots, q$ are real positive numbers, $a_{j}, j=1,2, \ldots, p$ and $b_{j}, j=1,2, \ldots, q$ are complex numbers, $L$ is a contour separating the poles of $\Gamma\left(b_{j}+\beta_{j} s\right), j=1,2, \ldots, m$ from those of $\Gamma\left(1-a_{j}-\alpha_{j} s\right), j=1,2, \ldots, n$. When $\alpha_{1}=1=\cdots=\alpha_{p}=\beta_{1}=1=\cdots=\beta_{q}$, then the $H$-function reduces to Meijer's $G$ - function, for more details see [18]. In a similar way we can evaluate the Laplace transform of the pathway model for $\alpha<1$ and is given by

$$
\begin{aligned}
L_{f_{1}}(t) & =c_{1} \int_{0}^{\left[\frac{1}{a(1-\alpha)}\right]^{\frac{1}{\delta}}} \mathrm{e}^{-t x} x^{\gamma_{1}}\left[1-a(1-\alpha) x^{\delta}\right]^{\frac{\eta}{1-\alpha}} \mathrm{d} x \\
& =\frac{\Gamma\left(1+\frac{\eta}{1-\alpha}+\frac{\gamma}{\delta}\right)}{\Gamma\left(\frac{\gamma}{\delta}\right)} H_{1,2}^{1,1}\left[\left.\frac{t}{a^{\frac{1}{\delta}}(1-\alpha)^{\frac{1}{\delta}}}\right|_{(0,1),\left(-\frac{\eta}{1-\alpha}-\frac{\gamma}{\delta}, \frac{1}{\delta}\right)} ^{\left(1-\frac{\gamma}{\delta}, \frac{1}{\delta}\right)}\right], \quad \Re(\gamma)>0
\end{aligned}
$$

THEOREM 1. For $\Re(\gamma)>0, \Re\left(\frac{\eta}{\alpha-1}-\frac{\gamma}{\delta}\right)>0, \delta>0, \eta>0, x>0, \alpha>1$, the Laplace transform (or the corresponding moment generating function) of the pathway model of the form $f_{2}(x)$, given in Euqation (12) goes to the Laplace transform (moment generating function) of the generalized gamma density given in Euqation (15) when $\alpha \rightarrow 1_{+}$.

THEOREM 2. For $\Re(\gamma)>0, \delta>0, \eta>0, x>0, \alpha<1$, the Laplace transform (or the corresponding moment generating function) of the pathway model of the form $f_{2}(x)$, given in Euqation (14) goes to the Laplace transform (moment generating function) of the generalized gamma density given in Euqation (15) when $\alpha \rightarrow 1_{-}$.

The limiting case is given by

$$
\begin{aligned}
L_{f}(t) & =c \int_{0}^{\infty} e^{-t x} x^{\gamma} e^{-b x^{\delta}} \mathrm{d} x \\
& =\frac{1}{\Gamma\left(\frac{\gamma}{\delta}\right)} H_{1,1}^{1,1}\left[\left.\frac{t}{b^{\frac{1}{\delta}}}\right|_{(0,1)} ^{\left(1-\frac{\gamma}{\delta}, \frac{1}{\delta}\right)}\right]
\end{aligned}
$$


The Laplace transform of this density also provides the moment generating function of the extended gamma density thereby the moment generating functions of extended form of Weibull, chisquare, Reyleigh, Maxwell-Boltzmann, exponential and other densities in this general class. Usually we do not find the moment generating function or Laplace transform and the characteristic function of the generalized gamma density in the literature when $\delta \neq 1$. Here we obtained the Laplace transform of the generalized gamma density, besides giving an extension to this density.

\subsection{Multivariate Generalizations}

One generalization of the model in Euqation (1) for one scalar case is given by

$$
\begin{aligned}
f_{1}\left(x_{1}, x_{2}, \cdots, x_{n}\right)= & K x_{1}^{\gamma_{1}-1} x_{2}^{\gamma_{2}-1} \cdots x_{n}^{\gamma_{n}-1} \\
& \times\left[1-(1-\alpha)\left(a_{1} x_{1}^{\delta_{1}}+a_{2} x_{2}^{\delta_{2}}+\cdots+a_{n} x_{n}^{\delta_{n}}\right)\right]^{\frac{\eta}{1-\alpha}}
\end{aligned}
$$

$\alpha<1, \eta>0, a_{i}>0, \delta_{j}>0, i=1,2, \cdots, n, 1-(1-\alpha) \sum_{i=1}^{n} a_{i} x_{i}^{\delta_{i}}>0$. We can see that Equation (16) is the Dirichlet family of densities. For $\alpha<1$, Equation (16) stays in the type-1 Dirichlet form and for $\alpha>1$ it stays as a type-2 Dirichlet form. This multivariate analogue can also produce multivariate extensions to Tsallis statistics and superstatistics. Here the variables are not independently distributed, but when $\alpha \rightarrow 1$ we have a surprising result that $x_{1}, x_{2}, \cdots, x_{n}$ will become independently distributed generalized gamma variables. Various generalizations of the pathway model are considered by Mathai and associates. The normalizing constants can be obtained by integrating out the variables one at a time, starting from $x_{n}$ an going to $x_{1}$ (see [23]).

\section{Connections to Astrophysics and Statistical Mechanics}

\subsection{Superstatistics Consideration and Pathway Model}

Beck and Cohen [3] developed the concept of superstatistics in statistical mechanics. From a statistical point of view, the procedure is equivalent to starting with a conditional density for a random variable $x$ at a given value of a parameter $\theta$. Then assume the parameter $\theta$ has a prior density. Consider the conditional density of the form

$$
f_{x \mid \theta}(x \mid \theta)=k_{1} x^{\gamma} \mathrm{e}^{-\theta x^{\delta}}, \gamma+1>0, \theta>0, \delta>0, x>0
$$

where $k_{1}=\frac{\delta \theta^{\frac{\gamma+1}{\delta}}}{\Gamma\left(\frac{\gamma+1}{\delta}\right)}$. Suppose that $\theta$ has an exponential density given by $f_{\theta}(\theta)=\lambda \mathrm{e}^{-\lambda \theta}$, $\lambda>0, \theta>0$. Then the unconditional density of $x$ is given by

$$
\begin{aligned}
f_{x}(x) & =\int_{\theta} f_{x \mid \theta}(x \mid \theta) f_{\theta}(\theta) \mathrm{d} \theta \\
& =\frac{\delta \Gamma\left(\frac{\gamma+1}{\delta}+1\right) x^{\gamma}\left[1+\frac{x^{\delta}}{\lambda}\right]^{-\left(\frac{\gamma+1}{\delta}+1\right)}}{\lambda^{\frac{\gamma+1}{\delta}} \Gamma\left(\frac{\gamma+1}{\delta}\right)}
\end{aligned}
$$

(see [2,3,11,18,24,25]). Equation (18) is the superstatistics of Beck and Cohen [3], in the sense of superimposing another distribution or the distribution of $x$ with superimposed distribution of the 
parameter $\theta$. In a physical problem the parameter $\theta$ may be something like temperature having its own distribution. Several physical interpretations of superstatistics are available from the papers of Beck and others. The factor $\left[1+\frac{x^{\delta}}{\lambda}\right]^{-\left(\frac{\gamma+1}{\delta}+1\right)}$ written as $\left[1+(\alpha-1) x^{\delta}\right]^{-\frac{1}{\alpha-1}}, \alpha>1$ is the foundation for the current hot topic of Tsallis statistics in non-extensive statistical mechanics. Observe that only a form of the type $\left[1+\frac{x^{\delta}}{\lambda}\right]^{-\left(\frac{\gamma+1}{\delta}+1\right)}$ where $1+\frac{x^{\delta}}{\lambda}>0, \lambda>0, x^{\delta}>0, \frac{\gamma+1}{\delta}+1>0$, that is, only a type- 2 beta form can come from such a consideration. In other words a type-1 beta form cannot come because for the convergence of the integral in Euqation (18), $a+x^{\delta}$ must be positive with $\lambda>0$ and $x^{\delta}>0$. It is to be pointed out here that the superstatistics of Beck and Cohen [2] and Beck [3] are available from the procedure given above. It goes without saying that only type-2 beta form as given in Euqation (2) is available from superstatistics considerations, see [26]. The conditional density of the random variable $\theta$, given $x$ is the posterior probability density of $\theta$ and is given by

$$
\begin{aligned}
f_{\theta \mid x}(\theta \mid x) & =\frac{f_{\theta}(\theta) f_{x \mid \theta}(x \mid \theta)}{f_{x}(x)} \\
& =\frac{\lambda^{\frac{\gamma+1}{\delta}+1}\left[1+\frac{x^{\delta}}{\lambda}\right]^{\frac{\gamma+1}{\delta}+1}}{\Gamma\left(\frac{\gamma+1}{\delta}+1\right)} \mathrm{e}^{-\theta\left(\lambda+x^{\delta}\right)} \theta^{\frac{\gamma+1}{\delta}}, \theta>0
\end{aligned}
$$

With the help of Euqation (19) we can obtain the Bayes' estimate of the parameter $\theta$. To this extent, let

$$
\begin{aligned}
\Phi(x) & =E_{\theta \mid x}(\theta \mid x)=\int_{0}^{\infty} \theta f_{\theta \mid x}(\theta \mid x) \mathrm{d} \theta \\
& =\frac{\frac{\gamma+1}{\delta}+1}{\lambda+x^{\delta}}
\end{aligned}
$$

Superstatistics and Tsallis statistics in statistical mechanics are given interpretations in terms of Bayesian statistical analysis. Subsequently superstatistics is extended by replacing each component of the conditional and marginal densities by Mathai's pathway model and further both components are replaced by Mathai's pathway model. This produces a wide class of mathematically and statistically interesting functions for prospective applications in statistical physics, see [19]. The same procedure can be used to look at the extended forms. Let the conditional density of $x$, given $\theta$, be of the form

$$
f_{\alpha_{x \mid \theta}}(x \mid \theta)=k_{2} x^{\gamma}\left[1+\theta(\alpha-1) x^{\delta}\right]^{-\frac{1}{\alpha-1}}, x>0, \theta>0, \alpha>1, \delta>0
$$

where $k_{2}=\frac{\delta(\theta(\alpha-1))^{\frac{\gamma+1}{\delta}} \Gamma\left(\frac{1}{\alpha-1}\right)}{\Gamma\left(\frac{\gamma+1}{\delta}\right) \Gamma\left(\frac{1}{\alpha-1}-\frac{\gamma+1}{\delta}\right)}$ and assume that the parameter $\theta$ has a prior density $f_{\theta}(\theta)=\lambda \mathrm{e}^{-\lambda \theta}, \lambda>$ $0, \theta>0$. Then the unconditional density of $x$ is given by

$$
f_{\alpha_{x}}(x)=\frac{\lambda \delta x^{-(\delta+1)}}{(\alpha-1) \Gamma\left(\frac{\gamma+1}{\delta}\right) \Gamma\left(\frac{1}{\alpha-1}-\frac{\gamma+1}{\delta}\right)} G_{1,2}^{2,1}\left[\left.\frac{\lambda}{x^{\delta}(\alpha-1)}\right|_{0, \frac{1}{\alpha-1}-\frac{\gamma+1}{\delta}-1} ^{-\frac{\gamma+1}{\delta}}\right], x>0
$$

where $G_{1,2}^{2,1}(\cdot)$ is a $G$-function. For the theory and application of the functions see Mathai [7]. The posterior probability density is given by

$$
f_{\alpha_{\theta \mid x}}(\theta \mid x)=c_{4}^{-1}(\alpha-1)^{\frac{\gamma+1}{\delta}+1} x^{\gamma+\delta+1} \Gamma\left(\frac{1}{\alpha-1}\right) \theta^{\frac{\gamma+1}{\delta}} \mathrm{e}^{-\lambda \theta}\left[1+\theta(\alpha-1) x^{\delta}\right]^{-\frac{1}{\alpha-1}}
$$


where $c_{4}=G_{1,2}^{2,1}\left[\left.\frac{\lambda}{x^{\delta}(\alpha-1)}\right|_{0, \frac{1}{\alpha-1}-\frac{\gamma+1}{\delta}-1} ^{-\frac{\gamma+1}{\delta}}\right]$. Then the Bayes' estimate of $\theta$, at given $x$, defined by $\Phi_{\alpha}(x)$, is given by the following:

$$
\Phi_{\alpha}(x)=E_{\alpha_{\theta \mid x}}(\theta \mid x)=\frac{c_{4}^{-1}}{(\alpha-1) x^{\delta}} G_{1,2}^{2,1}\left[\left.\frac{\lambda}{x^{\delta}(\alpha-1)}\right|_{0, \frac{1}{\alpha-1}-\frac{\gamma+1}{\delta}-2} ^{1-\frac{\gamma+1}{\delta}}\right]
$$

LEMMA 1. Let the conditional density of $x$ given $\theta$ be $f_{x \mid \theta}(x \mid \theta)$ as in Equation (17) and assume that the parameter $\theta$ has a prior density $f_{\theta}(\theta)=\lambda \mathrm{e}^{-\lambda \theta}, \lambda>0, \theta>0$. Then the Bayes' estimate of $\theta$ is given by $\Phi(x)$ in Equation (20).

THEOREM 3. Let the conditional density of $x$ given $\theta$ be $f_{\alpha_{x \mid \theta}}(x \mid \theta)$ as in Equation (21) and assume that the parameter $\theta$ has a prior density $f_{\theta}(\theta)=\lambda \mathrm{e}^{-\lambda \theta}, \lambda>0, \theta>0$. Then the Bayes' estimate of $\theta$ is given by $\Phi_{\alpha}(x)$ in Equation (24).

Thus, the popular superstatistics in statistical mechanics can be considered as a special case of the pathway model in Equation (18) for $\eta=1$ and $\delta=1$.

\section{2. $\alpha$-gamma Models Associated with Bessel Function}

Sebastian [27] deals with a new family of statistical distributions associated with Bessel function which gives an extension of the gamma density, which will connect the fractional calculus and statistical distribution theory through the special function. The idea is motivated by the fact that a non-central chi-square density is associated with a Bessel function. In order to make thicker or thinner tails in a gamma density we consider a density function of the following type:

$$
f_{x \mid a}(x \mid a)=\frac{\rho a^{\frac{\gamma}{\rho}} \mathrm{e}^{-\frac{\delta}{a}}}{\Gamma\left(\frac{\gamma}{\rho}\right)} x^{\gamma-1} \mathrm{e}_{0}^{-a x^{\rho}} F_{1}\left(; \frac{\gamma}{\rho} ; \delta x^{\rho}\right) ; 0<x<\infty
$$

where ${ }_{0} F_{1}(; b ; x)=\sum_{k=0}^{\infty} \frac{(x)^{k}}{(b)_{k} k !},(b)_{k}$ is the Pochhammer symbol, $(b)_{m}=b(b+1) \cdots(b+m-$ $1), b \neq 0,(b)_{0}=1$. When $\delta=0$ the Equation (25) reduces to generalized gamma density. Note that this is the generalization of some standard statistical densities such as gamma, Weibull, exponential, Maxwell-Boltzmann, Rayleigh and many more. When $\delta=0, \rho=2$, Equation (25) reduces to folded standard normal density. We can extend the generalized gamma model associated with Bessel function in Equation (25) by using the pathway model of Mathai [1], when $\alpha<1$ we get the extended function as

$$
\begin{aligned}
g_{\alpha}(x)= & k_{1} x^{\gamma-1}\left[1-a(1-\alpha) x^{\rho}\right]^{\frac{1}{1-\alpha}}{ }_{0} F_{1}\left(; \frac{\gamma}{\rho} ; \delta x^{\rho}\right) ; \alpha<1, \\
& a>0, \rho>0,1-a(1-\alpha) x^{\rho}>0, x>0
\end{aligned}
$$

where $k_{1}$ is the normalizing constant. Note that $g_{\alpha}(x)$ is a generalized type- 1 beta model associated with Bessel function. Observe that for $\alpha>1$, writing $1-\alpha=-(\alpha-1)$ in Equation (25) produces extended type- 2 beta form which is given by

$$
f_{\alpha}(x)=k_{2} x^{\gamma-1}\left[1+a(\alpha-1) x^{\rho}\right]^{-\frac{1}{\alpha-1}} 0 F_{1}\left(; \frac{\gamma}{\rho} ; \delta x^{\rho}\right) ; \alpha>1, a>0, \rho>0
$$


where $k_{2}$ is the normalizing constant. Note that in both the cases, when $\alpha \rightarrow 1$, we have Equation (25) and hence it can be considered to be an extended form of Equation (25). This model has wide potential applications in the discipline physical science especially in statistical mechanics, see [27,28]. For fixed values of $\gamma=2, \rho=1.2$ and $a=1$, we can look at the graphs for $\delta=-0.5, q<1, \delta=0.5, q<1$ as well as for $\delta=-0.5, q>1, \delta=0.5, q>1$. From the Figures 2, we can see that when $q$ moves from $-\infty$ to 1 , the curve becomes less peaked. Similarly from Figure 3, we can see that when $q$ moves from 1 to $\infty$, the curve becomes less peaked. It is also observed that when $\delta>0$ the right tail of the density becomes thicker and thicker. Similarly when $\delta<0$ the right tail gets thinner and thinner. Densities exhibiting thicker or thinner tail occur frequently in many different areas of science. For practical purposes of analyzing data from physical experiments and in building up models in statistics, we frequently select a member from a parametric family of distributions.

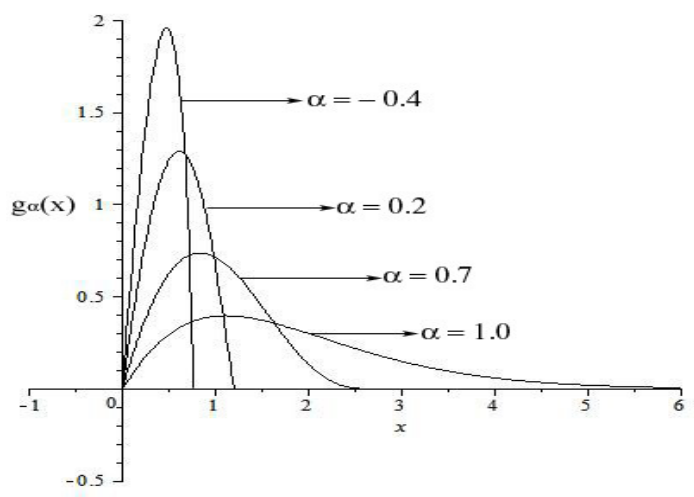

Figure 2. (Left) $\alpha$ gamma bessel model for $\delta=0.5, \alpha<1$; for $\delta=-0.5, \alpha<1$.

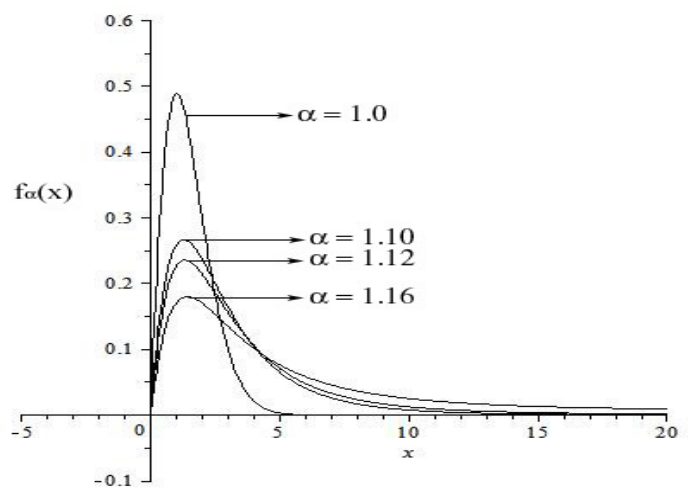

Figure 3. (Left) $\alpha$ gamma bessel model for $\delta=0.5, \alpha>1$; for $\delta=-0.5, \alpha>1$.

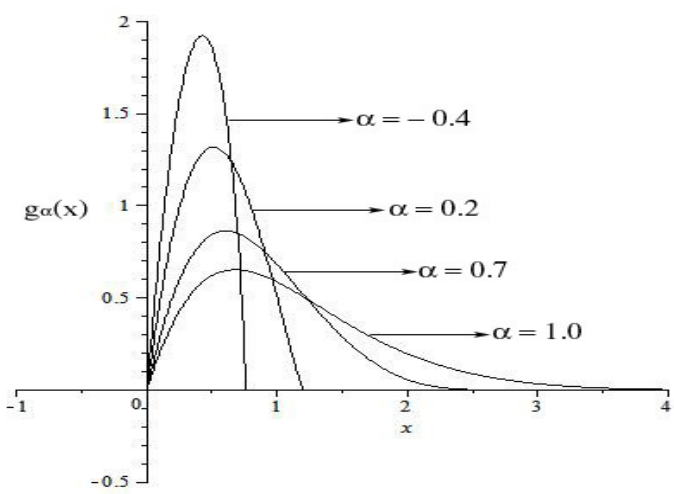

(Right) $\alpha$ gamma bessel model

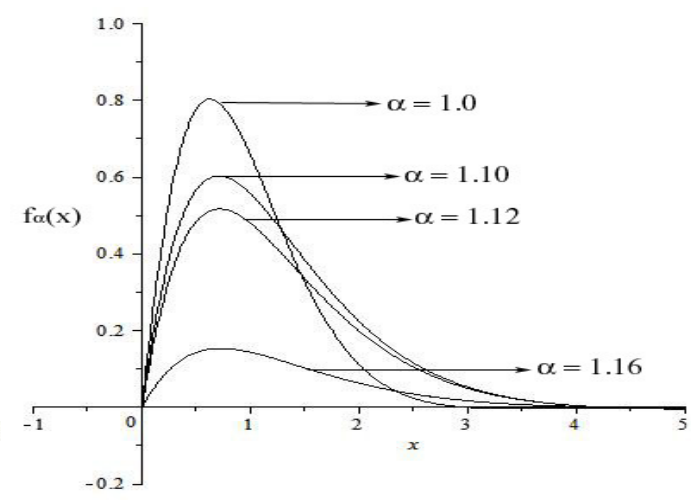

(Right) $\alpha$ gamma bessel model

\subsection{Tsallis Statistics}

Model Equation (1) for $\gamma=1, \delta=1, a=1, \eta=1$ is Tsallis statistics, which is the foundation for the newly created hot topic of non-extensive statistical mechanics. It is stated that over three thousand papers are written on Tsallis statistics so far. With $\alpha$ replaced in $-\alpha$ in Model Equation (1), with $\gamma=0, a=1, \eta=1, \delta=1$, one has an extension of the exponential function, known as $q$-exponential 
function [The parameter $q$ is used instead of $\alpha$ and hence $q$-exponential]. The basis for the current hot topic of $q$-calculus is this $q$-exponential function. When $\gamma=1, a=1, \eta=1, \delta=1$ one has the following property if the resulting function of $f(x)$ is divided by the resulting normalizing factor $c$ and obtain the function $g(x)$, that is, $g(x)=\frac{f(x)}{c}$. Then

$$
\frac{\mathrm{d} g(x)}{\mathrm{d} x}=-[g(x)]^{\alpha} .
$$

This is the power law in physics literature. Thus, power function law is a special case of the pathway model of Equations (1) and (2) for $\gamma=1, \delta=1, a=1, \eta=1$ and exactly the normalizing constants $c_{1}$ and $c_{2}$. Also Tsallis statistics can be looked upon as a special case of Equations (1) and (2) for $\gamma=1, \delta=1, a=1, \eta=1$.

\subsection{Extension of Thermonuclear Functions through Pathway Model}

Nuclear reactions govern major aspects of the chemical evolution of the universe. A proper understanding of the nuclear reactions that are going on in hot cosmic plasmas, and those in the laboratories as well, requires a sound theory of nuclear-reaction dynamics. The reaction probability integral is the probability per unit time that two particles, confined to a unit volume, will react with each other. Practically all applications of fusion plasmas are controlled in some way or other by the theory of thermonuclear reaction rates under specific circumstances. After several decades of effort, a systematic and complete theory of thermonuclear reaction rates has been developed [29-32].

The standard thermonuclear function in the Maxwell-Boltzmann case in the theory of nuclear reactions, is given by the following ([31,33]):

Nonresonant Case with Depleted Tail:

$$
I_{1}=\int_{0}^{\infty} x^{\gamma-1} e^{-a x^{\delta}-b x^{-\rho}} \mathrm{d} x, a>0, b>0, \delta>0, \rho>0
$$

Nonresonant case with depleted tail and high energy cut-off:

$$
I_{2}=\int_{0}^{d} x^{\gamma-1} e^{-a x^{\delta}-b x^{-\rho}} \mathrm{d} x, a>0, b>0, \delta>0, \rho>0, d<\infty
$$

Note that if $\delta=1$ is taken as the standard Maxwell-Boltzmannian behavior, then for $\delta>1$ the right tail will deplete faster and if $\delta<1$ then the depletion will be slower in Equation (29). We can extend the thermonuclear functions given in Equations (28) and (29) to more general classes by replacing $\mathrm{e}^{-b x^{-\rho}}$ by a binomial factor $\left[1-b(1-\alpha) x^{-\rho}\right]^{\frac{1}{1-\alpha}}([13,34,35])$. Thus we consider the general class of reaction rate integrals

$$
\begin{gathered}
I_{1 \alpha}=\int_{0}^{\infty} x^{\gamma-1} \mathrm{e}^{-a x^{\delta}}\left[1+b(\alpha-1) x^{-\rho}\right]^{-\frac{1}{\alpha-1}} \mathrm{~d} x, a>0, b>0, \delta>0, \rho>0, \alpha>1 \\
I_{2 \alpha}=\int_{0}^{d} x^{\gamma-1} \mathrm{e}^{-a x^{\delta}}\left[1-b(1-\alpha) x^{-\rho}\right]^{\frac{1}{1-\alpha}} \mathrm{d} x, a>0, b>0, \delta>0, \rho>0, \alpha<1
\end{gathered}
$$

We can evaluate these extended integrals by using Mellin convolution property. Equation (30) can be looked upon as the Mellin convolution of two real positive scalar independently distributed random 
variables $x_{1}$ and $x_{2}$, where $x_{1}$ has a generalized gamma density and $x_{2}$ has an extended form of stretched exponential function. Make the transformation $u=x_{1} x_{2}$ and $v=x_{1}$, and then proceed as in the case of the evaluation of the Laplace transform of the pathway model, we can arrive at the value of the extended reaction rate integral as

$$
\begin{aligned}
I_{1 \alpha} & =\int_{0}^{\infty} \mathrm{e}^{-a x^{\delta}} x^{\gamma-1}\left[1+b(\alpha-1) x^{-\rho}\right]^{-\frac{1}{\alpha-1}} \mathrm{~d} x \\
& =\frac{1}{\rho \mu a^{\frac{\gamma}{\delta}} \Gamma\left(\frac{1}{\alpha-1}\right)} H_{1,2}^{2,1}\left[\left.a^{\frac{1}{\delta}}(b(\alpha-1))^{\frac{1}{\rho}}\right|_{\left(0, \frac{1}{\rho}\right),\left(\frac{\gamma}{\delta}, \frac{1}{\delta}\right)} ^{\left(1-\frac{1}{\alpha-1}, \frac{1}{\rho}\right)}\right], b=u^{\rho}
\end{aligned}
$$

Similarly we can evaluate Equation (31) by considering it as the Mellin convolution of exponential density and pathway model for $\alpha<1$. When we take the limit as $\alpha \rightarrow 1$, in Equation (32), we will get the value of the reaction rate integral given in Equations (28) and (29), as an $H$-function.

\subsubsection{Inverse Gaussian as a Particular Case of the Pathway Model}

Note that one form of the inverse Gaussian probability density function is given by

$$
g(x)=k x^{-\frac{3}{2}} \mathrm{e}^{-\frac{\lambda}{2}\left(\frac{x}{\mu^{2}}+\frac{1}{x}\right)}, \mu \neq 0, x>0, \lambda>0
$$

where $k$ is the normalizing constant (see [7]). Put $\gamma=-\frac{5}{2}, \delta=1, \rho=1, a=\frac{\lambda}{2 \mu^{2}}, b=\frac{\lambda}{2}$ in Equation (28), we can see that the inverse Gaussian density is the integrand in $I_{1}$. Hence $I_{1}$ in Equation (28) can be used to evaluate the moments of inverse Gaussian density. Hence the integrand in the extended integral $I_{1 \alpha}$ can be considered as the extended form of the inverse Gaussian density.

\subsubsection{An Interpretation of the Pathway Parameter $\alpha$}

Let us start with the pathway density in Equation (1) with $\eta=1$. For this to remain a density we need the condition $1-a(1-\alpha) x^{\delta}>0$ or when $x$ is positive then $0<x<\frac{1}{[a(1-\alpha)]^{\frac{1}{\delta}}}, \alpha<1$, or if we have the model in Equation (31) then

$$
-\frac{1}{[a(1-\alpha)]^{\frac{1}{\delta}}}<x<\frac{1}{[a(1-\alpha)]^{\frac{1}{\delta}}}, \alpha<1
$$

Outside this range, the density is zero. Thus for $x>0$ the right tail of the density is cut-off at $\frac{1}{[a(1-\alpha)]^{\frac{1}{\delta}}}$. If $d$ is this cut-off on the right then

$$
d=\frac{1}{[a(1-\alpha)]^{\frac{1}{\delta}}} \Rightarrow \alpha=1-\frac{1}{a d^{\delta}}, \text { for } \alpha<1
$$

As $\alpha$ moves closer to 1 then the cut-off point $d$ moves farther out and eventually when $\alpha \rightarrow 1$ then $d \rightarrow \infty$. In this case $\alpha$ is computed easily from the cut-off. Also the pathway parameter $\alpha$ can be estimated in terms of arbitrary moments, see for example [12]. 


\section{Pathway Model and Fractional Calculus}

When solving certain problems in reaction-diffusion, relaxation-oscillations, it is observed that the solution is obtained in terms of exponential function, see $[33,36]$. But if a fractional integration is under consideration then the residual reaction equation is given by

$$
N(t)=N_{0}-c_{0}^{\nu} D_{t}^{-\nu} N(t), \quad \nu>0
$$

where ${ }_{0} D_{t}^{-\nu}$ is the standard Riemann-Louville fractional integral operator, where $N(t)$ is the number density of the reacting particles and Equation (34) has the solution in terms of generalized Mittag-Leffler function. While fitting such a model, Mittag-leffler function will provide a better fit as compared to exponential function. If we consider the residual rate of change $c$, in Equation (34) is a random variable having a gamma type density

$$
g(c)=\frac{w^{\mu}}{\Gamma(\mu)} c^{\mu-1} \mathrm{e}^{-w c}, \quad w>0,0<c<\infty
$$

where $\mu>0, w, \mu$ are known and $\frac{\mu}{w}$ is the mean value of $c$. The residual rate of change may have small probabilities of it being too large or too small and the maximum probability may be for a medium range of values for the residual rate of change $c$. Equation (34) is the situation where the residual rate of change is such that the production rate dominates so that we have the form $-c^{\nu}, \nu>0, c>0$. If the destruction rate dominates then the constant will be of the form $c^{\nu}, \nu>0, c>0$. By solving Equation (34), we have the unconditional number density of the following form:

$$
N(t)=\frac{N_{0}}{\Gamma(\mu)} t^{\mu-1}\left(1+\frac{t^{\nu}}{w^{\nu}}\right)^{-(\gamma+1)}, \quad 0<t<\infty, w>0
$$

If we make the substitution $\gamma+1=\frac{1}{\alpha-1}, \alpha>1 \Rightarrow \gamma=\frac{\alpha-2}{\alpha-1}$ and $w^{-\nu}=b(\alpha-1), b>0$. Then we have

$$
N(t)=\frac{N_{0}}{\Gamma(\mu)} t^{\mu-1}\left[1+b(\alpha-1) t^{\nu}\right]^{-\frac{1}{\alpha-1}}
$$

for $\alpha>1, t>0, b>0, \mu>0$. For general values of $\mu$ and $\alpha>1$ such that $\frac{1}{\alpha-1}-\frac{\mu}{\nu}>0$, Euqation (37) corresponds to the pathway model of Mathai as well as the superstatistics of Beck and Cohen [3]. As an application of pathway model in fractional calculus, a general pathway fractional integral operator is introduced in [37], which generalizes the classical Riemann-Liouville fractional integration operator, see Section 4.5.

\subsection{P-Transform}

Consider the generalized Krätzel function $D_{\rho, \beta}^{\nu, \alpha}(x)$, dealt with in [38], given by

$$
D_{\rho, \beta}^{\nu, \alpha}(x)=\int_{0}^{\infty} y^{\nu-1}\left[1+a(\alpha-1) y^{\rho}\right]^{-\frac{1}{\alpha-1}} \mathrm{e}^{-x y^{-\beta}} \mathrm{d} y, x>0
$$

with $\gamma \in \mathbb{C}, \beta>0, \alpha>1$. The generalized Krätzel function is obtained by using the pathway model introduced by Mathai [1]. The $\mathcal{P}$-transform or pathway transform introduced in [39] by using the pathway idea is defined as

$$
\left(\mathcal{P}_{\nu}^{\rho, \beta, \alpha} f\right)(x)=\int_{0}^{\infty} D_{\rho, \beta}^{\nu, \alpha}(x t) f(t) \mathrm{d} t, x>0
$$


where $D_{\rho, \beta}^{\nu, \alpha}(x)$ denotes the function given in Equation (38). The $\mathcal{P}$-transform is defined in the space $\mathbb{L}_{\nu, r}$ consisting of the lebesgue measurable complex valued functions $f$ for which

$$
\|f\|_{\nu, r}=\left\{\int_{0}^{\infty}\left|t^{\nu} f(t)\right|^{r} \frac{\mathrm{d} t}{t}\right\}^{\frac{1}{r}}<\infty,
$$

for $1 \leq r<\infty, \nu \in \mathbb{R}$. When $\beta=1, a=1$ and $\alpha \rightarrow 1$ the $\mathcal{P}$-transform reduces to the Krätzel tranform, introduced by Krätzel [40], which is given by

$$
\mathcal{K}_{\nu}^{(\rho)} f(x)=\int_{0}^{\infty} Z_{\rho}^{\nu}(x t) f(t) \mathrm{d} t, x>0
$$

where

$$
Z_{\rho}^{\nu}(x)=\int_{0}^{\infty} y^{\nu-1} \mathrm{e}^{-y^{\rho}-x y^{-1}} \mathrm{~d} y
$$

The $\mathcal{P}$-transform reduces to the Meijer transform when $\rho=1$ and $\alpha \rightarrow 1$. When $a=1, \beta=1$ and $\alpha \rightarrow 1$, then the generalized Krätzel function defined in Equation (38) reduces to the modified Bessel function of the third kind or McDonald function ([38,41,42]) have considered Equation (38) for $\beta=1$ and established its composition with fractional operators and represented it in terms of various generalized special functions. The Krätzel function defined in Equation (42) for any real $\rho$, was studied by [43] and established its representations in terms of $H$-function and extended the function from positive $x>0$ to complex $z$. Here we establishe connection between generalized Krätzel function and $\mathcal{P}$-transform with generalized special functions.

The particular case of the kernel of the $\mathcal{P}$-transform given in Equation (38) is the extended non-resonant thermonuclear function used in Astrophysics which is already discussed. As $\alpha \rightarrow 1$ we get the standard reaction rate probability integral in the Maxwell-Boltzmann case. The behavior of the generalized Krätzel function Equation (38) can be studied from the following graphs. We take $a=1, \beta=1, \nu=2, \rho=3$ and $a=1, \beta=1, \nu=2, \rho=5$ for example and investigate the behavior of $D_{3,1}^{2, \alpha}(x)$ and $D_{5,1}^{2, \alpha}(x)$ for various values of $\alpha>1$ and $\alpha \rightarrow 1$. The graphs of these functions $D_{3,1}^{2, \alpha}(x)$ and $D_{5,1}^{2, \alpha}(x)$ at $\alpha=1, \alpha=1.25, \alpha=1.5, \alpha=1.8$ are given in Figure 4a,b, respectively. From these graphs we observe that if the value of the parameter $\alpha$ increases then the curves move away from the limiting situation for $\alpha \rightarrow 1$.
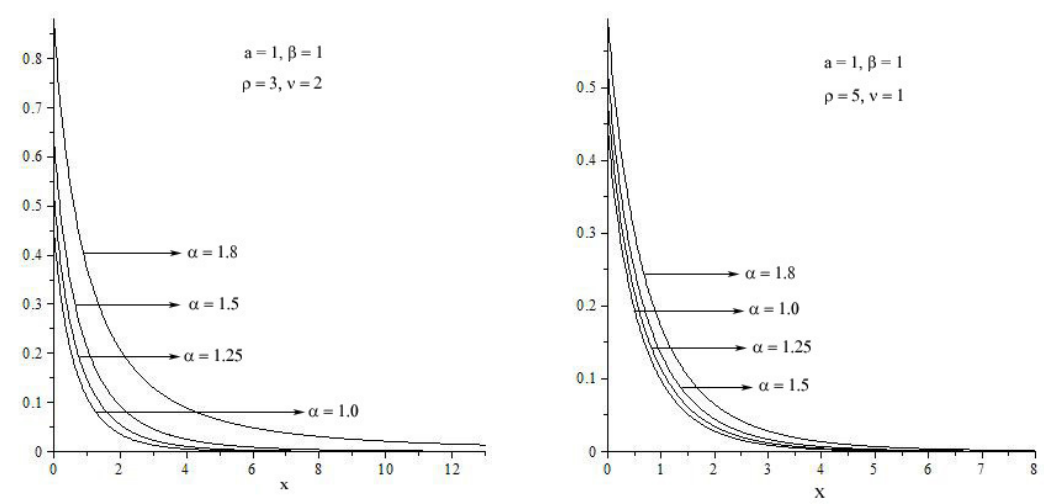

Figure 4. (Left) Behaviour of $D_{3,1}^{2, \alpha}(x)$ for various values of $\alpha>1$ and $\alpha \rightarrow 1$; (Right) Behaviour of $D_{5,1}^{2, \alpha}(x)$ for various values of $\alpha>1$ and $\alpha \rightarrow 1$. 


\section{A Matrix Variate Pathway Model}

Let $X=\left(x_{i j}\right), i=1, \ldots, p, j=1, \ldots, q, q \geq p$, of rank $p$ and of real scalar variables $x_{i j}^{\prime} s$ for all $i$ and $j$, subject to the condition that the rank of $X$ is $p$, having the density $f(X)$, where $f(X)$ is a scalar function of $X$ is given by

$$
f(X)=C\left|A^{\frac{1}{2}}(X-M) B(X-M)^{\prime} A^{\frac{1}{2}}\right|^{\gamma}\left|I-a(1-\alpha) A^{\frac{1}{2}}(X-M) B(X-M)^{\prime} A^{\frac{1}{2}}\right|^{\frac{\eta}{1-\alpha}}
$$

where $C$ is the normalizing constant, $A=A^{\prime}>0, B=B^{\prime}>0, A$ is $p \times p, B$ is $q \times q, A$ and $B$ are constant positive definite matrices, and $M$ is a $p \times q$ constant matrix. $A^{\frac{1}{2}}$ denotes the positive definite square root of $A, a>0$ and $\alpha$ is the pathway parameter. For keeping non-negativity of the determinant in Equation (43) we need the condition

$$
I-a(1-\alpha) A^{\frac{1}{2}}(X-M) B(X-M)^{\prime} A^{\frac{1}{2}}>0
$$

where, for example, the notation $A<Y<B \Rightarrow A=A^{\prime}>0, B=B^{\prime}>0, Y=Y^{\prime}>0, Y-A>$ $0, B-Y>0$. In Equation (43) the constant matrix $M$ can act as a relocation matrix or as the mean value matrix so that $E(X)=M$ where $E$ denotes the expected value.

\subsection{The Normalizing Constants}

This requires certain transformations and the knowledge of the corresponding Jacobians. Here we will use a few multi-linear and some nonlinear transformations and the corresponding Jacobians. The details of the derivations of these Jacobians are available from [44]. Put

$$
Y=A^{\frac{1}{2}}(X-M) B^{\frac{1}{2}} \Rightarrow \mathrm{d} Y=|A|^{\frac{q}{2}}|B|^{\frac{p}{2}} \mathrm{~d} X
$$

where $|(\cdot)|$ denotes the determinant of $(\cdot), \mathrm{d} X$ is defined as the following wedge product of differentials:

$$
\mathrm{d} X=\mathrm{d} x_{11} \wedge \mathrm{d} x_{12} \wedge \cdots \wedge \mathrm{d} x_{1 q} \wedge \mathrm{d} x_{22} \wedge \cdots \wedge \mathrm{d} x_{2 q} \wedge \cdots \wedge \mathrm{d} x_{p q}
$$

see [44] for the Jacobian. Since $f(X)$ is assumed to be a density,

$$
1=\int_{X} f(X) \mathrm{d} X=C|A|^{-\frac{q}{2}}|B|^{-\frac{p}{2}} \int_{Y}\left|Y Y^{\prime}\right|^{\gamma}\left|I-a(1-\alpha) Y Y^{\prime}\right|^{\frac{\eta}{1-\alpha}} \mathrm{d} Y
$$

Now, make suitable transformations and integrating out over the Stiefel manifold (for details, see [1]) we have

$$
\begin{aligned}
1 & =\int_{X} f(X) \mathrm{d} X \\
& =C|A|^{-\frac{q}{2}}|B|^{-\frac{p}{2}} \frac{\pi^{\frac{p q}{2}}}{\Gamma_{p}\left(\frac{q}{2}\right)} \int_{Z}|Z|^{\gamma+\frac{q}{2}-\frac{p+1}{2}}|I-a(1-\alpha) Z|^{\frac{\eta}{1-\alpha}} \mathrm{d} Z
\end{aligned}
$$

where, for example,

$$
\Gamma_{p}(\beta)=\pi^{\frac{p(p-1}{4}} \Gamma(\beta) \Gamma\left(\beta-\frac{1}{2}\right) \ldots \Gamma\left(\beta-\frac{p-1}{2}\right), \Re(\beta)>\frac{p-1}{2}
$$


is the real matrix-variate gamma and $\Re(\cdot)$ denotes the real part of $(\cdot)$.

Further evaluation of the integral in Equation (44) depends on the value of $\alpha$. If $\alpha<1$ then Equation (44) belongs to the real matrix-variate type-1 beta. Then integrating out with the help of a matrix variate type-1 beta integral we get, for $q \geq p, \alpha<1$,

$$
C=|A|^{\frac{q}{2}}|B|^{\frac{p}{2}}[a(1-\alpha)]^{p\left(\gamma+\frac{q}{2}\right)} \frac{\Gamma_{p}\left(\frac{q}{2}\right) \Gamma_{p}\left(\gamma+\frac{q}{2}+\frac{\eta}{1-\alpha}+\frac{p+1}{2}\right)}{\pi^{\frac{p q}{2}} \Gamma_{p}\left(\gamma+\frac{q}{2}\right) \Gamma_{p}\left(\frac{\eta}{1-\alpha}+\frac{p+1}{2}\right)}
$$

for $\alpha<1, \eta>0, a>0, \Re\left(\gamma+\frac{q}{2}\right)>\frac{p-1}{2}, q \geq p, p, q=1,2, \ldots, A=A^{\prime}>0, B=B^{\prime}>0$. For $\alpha>1$, write $1-\alpha=-(\alpha-1), \alpha>1$ then the integral in Equation (44) goes into a type-2 beta form. Then integrating out with the help of a real matrix-variate type-2 beta integral we have for $\alpha>1$,

$$
C=\frac{|A|^{\frac{q}{2}}|B|^{\frac{p}{2}}[a(\alpha-1)]^{p\left(\gamma+\frac{q}{2}\right)} \Gamma_{p}\left(\frac{q}{2}\right) \Gamma_{p}\left(\frac{\eta}{\alpha-1}\right)}{\pi^{\frac{p q}{2}} \Gamma_{p}\left(\gamma+\frac{q}{2}\right) \Gamma_{p}\left(\frac{\eta}{\alpha-1}-\gamma-\frac{q}{2}\right)}
$$

for $\alpha>1, \Re\left(\gamma+\frac{q}{2}\right)>\frac{p-1}{2}, \Re\left(\frac{\eta}{\alpha-1}-\gamma-\frac{q}{2}\right)>\frac{p-1}{2}, a>0, \eta>0, A=A^{\prime}>0, B=B^{\prime}>0$. When $\alpha \rightarrow 1$ we have

$$
\lim _{\alpha \rightarrow 1}|I-a(1-\alpha) Z|^{\frac{\eta}{1-\alpha}}=\mathrm{e}^{-a \eta \operatorname{tr}(Z)}
$$

and hence, evaluating with the help of a real matrix-variate gamma integral, we will get $C$ for $\alpha \rightarrow 1$,

$$
C=\frac{|A|^{\frac{q}{2}}|B|^{\frac{p}{2}}(a \eta)^{p\left(\gamma+\frac{q}{2}\right)} \Gamma_{p}\left(\frac{q}{2}\right)}{\pi^{\frac{p q}{2}} \Gamma_{p}\left(\gamma+\frac{q}{2}\right)}
$$

for $\Re\left(\gamma+\frac{q}{2}\right)>\frac{p-1}{2}, a>0, \eta>0$.

A model corresponding to Equation (43) in the complex domain, along with its properties and connections to various other fields, is studied in [23].

\subsection{Density of the Volume Content}

The squared volume of the $p$-parallelotope in the $q$-space,

$$
v^{2}=\left|A^{\frac{1}{2}}(X-M) B(X-M)^{\prime} A^{\frac{1}{2}}\right|
$$

The density of $u=v^{2}$ in Equation (49) can be evaluated by looking at the $h$-th moment of $v^{2}$ for an arbitrary $h$. That is, the expected value of $\left(v^{2}\right)^{h}$, is given by

$$
E\left(v^{2}\right)^{h}=\int_{X}\left(v^{2}\right)^{h} f_{5}(X) \mathrm{d} X
$$

This is available from the normalizing constants in Equations (46)-(48) by observing that the only change is that the parameter $\gamma$ is changed to $\gamma+h$. Hence from Equation (46) for $\alpha<1$,

$$
E\left(v^{2}\right)^{h}=[a(1-\alpha)]^{-p h} \frac{\Gamma_{p}\left(\gamma+\frac{q}{2}+h\right)}{\Gamma_{p}\left(\gamma+\frac{q}{2}\right)} \frac{\Gamma_{p}\left(\gamma+\frac{q}{2}+\frac{\eta}{1-\alpha}+\frac{p+1}{2}\right)}{\Gamma_{p}\left(\gamma+\frac{q}{2}+\frac{\eta}{1-\alpha}+\frac{p+1}{2}+h\right)}
$$


for $\alpha<1, \Re\left(\gamma+\frac{q}{2}\right)>\frac{p-1}{2}, \Re\left(\gamma+\frac{q}{2}+h\right)>\frac{p-1}{2}, \eta>0, a>0$. Let $u_{1}=[a(1-\alpha)]^{p} v^{2}$. Then

$$
\begin{aligned}
E\left(u_{1}^{h}\right) & =\prod_{j=1}^{p} \frac{\Gamma\left(\gamma+\frac{q}{2}-\frac{j-1}{2}+h\right)}{\Gamma\left(\gamma+\frac{q}{2}-\frac{j-1}{2}\right)} \frac{\Gamma\left(\gamma+\frac{q}{2}+\frac{\eta}{1-\alpha}+\frac{p+1}{2}-\frac{j-1}{2}\right)}{\Gamma\left(\gamma+\frac{q}{2}+\frac{\eta}{1-\alpha}+\frac{p+1}{2}-\frac{j-1}{2}+h\right)} \\
& =E\left(v_{1}^{h}\right) E\left(v_{2}^{h}\right) \ldots E\left(v_{p}^{h}\right)
\end{aligned}
$$

where $v_{j}$ is a real scalar type- 1 beta random variable with the parameters $\left(\gamma+\frac{q}{2}-\frac{j-1}{2}, \frac{\eta}{1-\alpha}+\frac{p+1}{2}\right), j=$ $1, \ldots, p$, and further, $v_{1}, \ldots, v_{p}$ are statistically independently distributed. Hence structurally

$$
u_{1}=v_{1} v_{2} \ldots v_{p}
$$

and the density of $u_{1}$ is that of $v_{1} \ldots v_{p}$. This is available by treating Equation (51) as coming from the Mellin transform of the density $g_{1}\left(u_{1}\right)$ of $u_{1}$. Even though the density $g_{1}\left(u_{1}\right)$ is unknown, its Mellin transform is available from Equation (51) for $h=s-1$. Hence from the unique inverse Mellin transform

$$
g_{1}\left(u_{1}\right)=u_{1}^{-1} \frac{1}{2 \pi i} \int_{L}\left[E\left(u_{1}^{h}\right)\right] u_{1}^{-h} \mathrm{~d} h
$$

where $i=\sqrt{-1}, L$ is a suitable contour and $E\left(u_{1}^{h}\right)$ is given in Equation (51). But the structure in Equation (51) is that of a Mellin transform of a G-function of the type $G_{p, p}^{p, 0}(\cdot)$. Hence

$$
\begin{aligned}
g_{1}\left(u_{1}\right) & =u_{1}^{-1}\left[\prod_{j=1}^{p} \frac{\Gamma\left(\gamma+\frac{q}{2}+\frac{\eta}{1-\alpha}+\frac{p+1}{2}-\frac{j-1}{2}\right.}{\Gamma\left(\gamma+\frac{q}{2}-\frac{j-1}{2}\right)}\right] \\
& \times G_{p, p}^{p, 0}\left[\begin{array}{l}
\frac{\eta}{1-\alpha}+\frac{p+1}{2}, j=1, \ldots, p \\
\gamma+\frac{q}{2}-\frac{j-1}{2}, j=1, \ldots, p
\end{array}\right], 0<u_{1}<1
\end{aligned}
$$

For $\alpha>1$, from Equation (47), $u_{2}=[a(\alpha-1)]^{p} v^{2}$ is a product of $p$ statistically independently distributed real scalar type- 2 beta random variables and hence the density of $u_{2}$ can be written in terms of a G-function of the type $G_{p, p}^{p, p}(\cdot)$.

Similarly for $\alpha \rightarrow 1, u_{3}=(a \eta)^{p} v^{2}$ is structurally a product of $p$ statistically independently distributed real gamma random variables and the density of $u_{3}$ can be written in terms of a G-function of the type $G_{0, p}^{p, 0}(\cdot)$.

A form such as the one in Equation (52) is connected to the $\lambda$-criterion in the likelihood ratio principle of testing statistical hypothesis on the parameters of one or more multivariate Gaussian populations. The pathway model for $\alpha<1$ can be structurally identified with a constant multiple of the one-to-one function of the $\lambda$-criterion in many situations in multivariate statistical analogues.

\subsection{Connection to Likelihood Ratio Criteria Consider the case $\alpha<1$.}

From Equations (51) and (52) one can see a structural representation in the form of a product of $p$ statistically independently distributed type- 1 beta random variables. Such a structure can also arise from a determinant of the type

$$
\lambda=\frac{\left|G_{1}\right|}{\left|G_{1}+G_{2}\right|}
$$

where $G_{1}$ and $G_{2}$ are independently distributed real matrix-variate gamma variables with the same scale parameter matrix. Such matrix representations are recently examined in [45]. A particular case of a 
real matrix-variate gamma matrix is a Wishart matrix. When $G_{1}$ and $G_{2}$ are independently distributed Wishart matrices $\lambda$ in Equation (54) corresponds to the likelihood ratio test criterion or a one-to-one function of it in multivariate statistical analysis. A large number of test criteria based on the principle of maximum likelihood have the structure in Equation (54), with a representation as in Equation (52) in the null case or when the statistical hypothesis is true. Distribution of the test statistic, when the null hypothesis is true, is known as the null distribution. Thus, a large number of null distributions, associated with the tests of hypotheses on the parameters of one or more multivariate normal populations, have the structure in Equation (54) and thus a large number of cases are covered by our discussion above. Hence a direct link can be established between the volume of a random $p$-parallelotope and the $\lambda$-criterion in Equation (54).

Various types of generalizations of the Dirichlet distribution are recently studied by [46-48]. In these papers there are several theorems characterizing or uniquely determining these generalized Dirichlet models through a product of independently distributed real scalar type-1 beta random variables. In all these cases one can establish a direct link from Equation (52) to Equation (54) providing explicit representations of the densities in terms of generalized Dirichlet integrals. As byproducts, these can also produce several results connecting G-functions and Dirichlet integrals. One such characterization theorem is recently explored by [49].

\subsection{Quadratic Forms}

For $p=1$ and $q>1$, Equation (49) gives

$$
Q=(X-M) B(X-M)^{\prime}
$$

the quadratic form.

Current theory of quadratic form in random variables is based on the assumption of a multivariate Gaussian population. But from Equation (43) we have a generalized quadratic form in $X$, where $X$ has the density in Equation (43), and the density of $Q$ is available from the step in Equation (44) to Equation (53). The theory of quadratic form in random variables can be extended to the samples from the density in Equation (43), rather than confining the study to Gaussian population. For the study of quadratic and bilinear forms see [15,23]. When $p=1$ the quadratic form in Equation (55) can be given many interpretations. Let $B=V^{-1}$ where $V$ is the covariance matrix in $X$, that is, $V=\operatorname{cov}(X)=$ $E\left[(X-\mu)^{\prime}(X-\mu)\right]$ where $X-\mu$ is $1 \times q, q>1$. Since $Y=V^{-\frac{1}{2}}(X-\mu)^{\prime}$ is the standardized $X$, $Y Y^{\prime}=y_{1}^{2}+\ldots+y_{q}^{2}=(X-\mu) V^{-1}(X-\mu)^{\prime}$ is the square of the generalized distance between $X$ and $\mu$. Also $(X-\mu) V^{-1}(X-\mu)^{\prime}=c>0$ is the ellipsoid of concentration in $X$ or a scalar measure of the extent of dispersion in $X-\mu$. The components in $X$ can be given physical interpretations. Consider a growing and moving rain droplet in a tropical cloud. Then $x_{1}$ could be the surface area, $x_{2}$ could be the energy content, $x_{3}$ the velocity in a certain direction and so on. The components in this case have joint variations. When $p=1$ one can derive the pathway density in Equation (43) through an optimization of a certain measure of entropy also.

\subsection{Pathway Fractional Integral}


Recently, an extension of classical fractional integral operators of scalar functions of scalar variables to the matrix-variate cases has been given by [50]. Real-valued scalar functions of matrix argument, where the argument matrix is real and positive definite, are used in the extensions. In this regard, a matrix-variate pathway fractional integral operator is introduced, see [51] which may be regarded as a generalization of matrix-variate Riemann-Liouville fractional integral operator. Moreover, from this operator one can figure out all the matrix-variate fractional integrals and almost all the extended densities for the pathway parameter $\alpha<1$ and $\alpha \rightarrow 1$. Through this new fractional integral operator, one can go to matrix-variate gamma to matrix-variate Gaussian or normal density with appropriate parametric values. In the present paper we bring out the idea of matrix-variate pathway to the corresponding fractional integral transform. Consequently a scalar version of pathway fractional integral operator can also be deduced, which generalizes the classical Reimann-Liouville fractional integration operator. The pathway fractional integral operator has found applications in reaction-diffusion problems, non-extensive statistical mechanics, non-linear waves, fractional differential equations, non-stable neighborhoods of physical system etc.

The following definition and notation is given for the matrix-variate pathway fractional integral:

$$
\left(P_{O+}^{(\eta, \alpha)} f\right)(X)=|X|^{\eta-\frac{p+1}{2}} \int_{T}\left|I-a(1-\alpha) X^{-\frac{1}{2}} T X^{-\frac{1}{2}}\right|^{\frac{\eta}{(1-\alpha)}-\frac{p+1}{2}} f(T) \mathrm{d} T
$$

where $O$ stands for a null matrix, and $T=T^{\prime}>0$ and $O$ are $p \times p$ matrices and $f(T)$ is a real-valued integrable scalar function of $T$ and $\mathrm{d} T$ is the wedge product of differentials. Also $a, \alpha$ scalars, $\eta \in C$ $a>0, I-a(1-\alpha) X^{-\frac{1}{2}} T X^{-\frac{1}{2}}>0$ (positive definite) and $\alpha$ is the pathway parameter, $\alpha<1$. It is hoped that the matrix-variate extensions of the operator will enable researchers working in physical, chemical and engineering sciences to extend their theories to the corresponding matrix-variate situations.

When $\alpha \rightarrow 1_{-},\left|I-a(1-\alpha) X^{-\frac{1}{2}} T X^{-\frac{1}{2}}\right|^{\frac{\eta}{(1-\alpha)}-\frac{P+1}{2}} \rightarrow \mathrm{e}^{-a \eta \operatorname{tr}\left[X^{-\frac{1}{2}} T X^{-\frac{1}{2}}\right]}$. This follows from the following facts: Since $X^{-\frac{1}{2}} T X^{-\frac{1}{2}}$ is real symmetric there exists an orthogonal matrix $Q$ such that $Q^{\prime} X^{-\frac{1}{2}} T X^{-\frac{1}{2}} Q$ will be a diagonal matrix with eigenvalues being the diagonal elements. Then when the limit is applied, each factor goes to the exponent and sum up to equate to the trace. Thus the operator will become

$$
\left(P_{O+}^{(\eta, 1)} f\right)(X)=|X|^{\eta-\frac{P+1}{2}} \int_{T=T^{\prime}>0} \mathrm{e}^{-\operatorname{tr}\left[a \eta X^{-1} T\right]} f(T) \mathrm{d} T=|X|^{\eta-\frac{P+1}{2}} L_{f}\left(a \eta X^{-1}\right)
$$

When $\alpha=0, a=1$ in Equation (56), the integral will become,

$$
\begin{aligned}
\left(P_{O+}^{(\eta, 0)} f\right)(X) & =|X|^{\eta-\frac{P+1}{2}} \int_{O<T<X}\left|I-X^{-\frac{1}{2}} T X^{-\frac{1}{2}}\right|^{\eta-\frac{P+1}{2}} f(T) \mathrm{d} T, \Re(\eta)>\frac{p-1}{2} \\
& =\int_{O<T<X}|X-T|^{\eta-\frac{P+1}{2}} f(T) \mathrm{d} T=\Gamma_{p}(\eta)_{O} D_{X}^{-\eta} f
\end{aligned}
$$

where ${ }_{O} D_{X}^{-\eta}$ is the matrix-variate extension of the standard left-sided Reimann-Liouville fractional integral and is defined as

$$
{ }_{O} D_{X}^{-\eta} f=\frac{1}{\Gamma_{p}(\eta)} \int_{O<T<X}|X-T|^{\eta-\frac{P+1}{2}} f(T) \mathrm{d} T, \quad \Re(\eta)>\frac{p-1}{2}
$$

When $p=1$ in Equation (56), one can obtain the scalar version of the pathway fractional integral operator, see [37] and is defined by the following: 
Let $f(x) \in L(a, b), \eta \in C, \Re(\eta)>0, a>0$ and $\alpha<1$, then

$$
\left(P_{0+}^{(\eta, \alpha)} f\right)(x)=x^{\eta-1} \int_{0}^{\left[\frac{x}{a(1-\alpha)}\right]}\left[1-\frac{a(1-\alpha) t}{x}\right]^{\frac{\eta}{(1-\alpha)}-1} f(t) \mathrm{d} t
$$

where $\alpha$ is the pathway parameter and $f(t)$ is an arbitrary function. In [37], it is shown that as $\alpha \rightarrow 1_{-}$, Equation (59) takes the form of Laplace transform of the arbitrary function $f(t)$. Again when $\alpha=0, a=$ 1, Equation (59) reduces to the left-sided Reimann-Liouville fractional integral operator. By the way an idea of thicker or thinner tail model associated with Mittag-Leffler function is obtained. As a result, generalized gamma Mittag-Leffler density can be obtained as a limiting case of pathway operator. It is also shown that under the conditions $\alpha=0, a=1$ and as $f(t)$ changes to ${ }_{2} F_{1}\left(\eta+\beta,-\gamma ; \eta ; 1-\frac{t}{x}\right) f(t)$, Equation (59) yields the Saigo fractional integral operator. Thus we can obtain all the generalizations, like [41,52,53], of left-sided fractional integrals by suitable substitutions, so that we call it the pathway fractional operator, a path through $\alpha$, leading to the above known fractional operators.

The importance of the operator is that a connection is established to wide classes of statistical distributions, to several types of situations in physics, chemistry, to the input-output situations in social sciences, in reaction-diffusion problems etc. The pathway parameter $\alpha$ establishes a path of going from one family of distributions to another family and to different classes of distributions. Thus, the pathway fractional operator, will enable us to derive a number of results covering wide range of distributions. The "fractional integration" nature of the operator will then extend the corresponding results to wider ranges, where when the pathway parameter $\alpha$ goes to 1 the corresponding results on generalized gamma type functions are obtained.

\section{Open Problem}

The main idea to introduce the pathway model is the switching property of the binomial function to the corresponding exponential function. That is

$$
\lim _{\alpha \rightarrow 1} F_{0}\left(\frac{1}{1-\alpha} ; ;-a(1-\alpha) x^{\delta}\right)=\lim _{\alpha \rightarrow 1}\left(1+a(1-\alpha) x^{\delta}\right)^{-\frac{1}{1-\alpha}}=\mathrm{e}^{-a x^{\delta}}, a>0
$$

Similar to Equation (60) we can develop a pathway connecting Bessel to exponential, as given below,

$$
\lim _{\alpha \rightarrow 1}{ }_{0} F_{1}\left(; \frac{1}{1-\alpha} ;-\frac{a x^{\delta}}{(1-\alpha)}\right)={ }_{0} F_{0}\left(; ;-a x^{\delta}\right)=\mathrm{e}^{-a x^{\delta}}, a>0
$$

Thus $\alpha$ can provide a pathway between Bessel functions and exponential functions. Thus, in all types of applications where Bessel functions are used, one can extend the scope of applications into an exponential form. In a physical system, if the exponential form gives the stable situation, then the parameter $\alpha$ will provide a pathway between stable and chaotic situations. So far, this area which offers a wide scope of possibilities, has yet to be explored.

\section{Acknowledgments}

Authors acknowledge gratefully the encouragement given by Professor A. M. Mathai, Department of Mathematics and Statistics, McGill University, Montreal, Canada H3A 2K6 and Professor H. J. Haubold, Office of Outer Space Affairs, United Nations, Vienna International Centre, Austria. 


\section{Conflicts of Interest}

The authors declare that there is no conflict of interests regarding the publication of this article.

\section{References}

1. Mathai, A.M. A Pathway to matrix-variate gamma and normal densities. Linear Algebra Appl. 2005, 396, 317-328.

2. Beck, C. Stretched exponentials from superstatistics. Phys. A 2006, 365, 96-101.

3. Beck, C.; Cohen, E.G.D. Superstatistics. Phys. A 2003, 322, 267-275.

4. Tsallis, C. Possible generalizations of Boltzmann-Gibbs statistics. J. Stat. Phys. 1988, 52, 479-487.

5. Tsallis, C. What should a statistical mechanics satisfy to reflect nature? Phys. D 2004, 193, 3-34.

6. Honerkamp, J. Stochastic Dynamical Systems: Concepts, Numerical Methods, Data Analysis; VCH Publishers: New York, NY, USA, 1994.

7. Mathai, A.M. A Handbook of Generalized Special Functions for Statistical and Physical Sciences; Clarendon Press: Oxford, UK, 1993.

8. Haubold, H.J.; Kumar, D.; Nair, S.S.; Joseph, D.P. Special functions and pathways for problems in astrophysics: An essay in honor of A.M. Mathai. Fract. Calc. Appl. Anal. 2010, 13, 133-158.

9. Joseph, D. P. Gamma distribution and extensions by using pathway idea. Stat. Pap. 2009, doi:10.1007/s00362-009-0231-y.

10. Mathai, A.M.; Rathie, P.N. Basic Concepts in Information Theory and Statistics: Axiomatic Foundations and Applications; Wiley Halsted: New York, NY, USA, 1975.

11. Mathai, A.M.; Haubold, H.J. On generalized entropy measures and pathways. Phys. A Stat. Mech. Appl. 2007, 385, 493-500.

12. Mathai, A.M.; Haubold, H.J. Pathway model Pathway model, superstatistics Tsallis statistics and a generalized measure of entropy. Phys. A 2007, 375, 110-122.

13. Mathai, A.M.; Haubold, H.J. Pathway parameter and thermonuclear functions. Phys. A Stat. Mech. Appl. 2008, 387, 2462-2470.

14. Mathai, A.M. On non-central generalized Laplacianness of quadratic forms in normal variables. J. Multivar. Anal. 1993, 45, 239-246.

15. Mathai, A.M.; Provost, S.B.; Hayakawa, T. Bilinear Forms and Zonal Polynomials, Lecture Notes; Springer-Verlag: New York, NY, USA, 1995.

16. Mathai, A.M. The residual effect of a growth-decay mechanism and the distributions of covariance structures. Can. J. Stat. 1993, 21, 277-283.

17. Mathai, A. M. Some properties of Mittag- Leffler functions and matrix variate analogues: A statistical perspective. Fract. Cal. Appl. Anal. 2010, 30, 113-132.

18. Mathai, A.M.; Saxena, R.K.; Haubold, H.J. The H-Function Theory and Applications; Springer: New York, NY, USA, 2010.

19. Mathai, A.M.; Haubold, H.J. A pathway from bayesian statistical analysis to superstatistics. 2010, arXiv:1011.5658v1. 
20. Mathai, A.M.; Moschopoulos, P. A Pathway Idea for Model Building. J. Stat. Appl. Proc. 2012, 1, $15-20$.

21. Pillai, R.N. On Mittag-Leffler functions and related distributions. Ann. Inst. Statist. Math. 1990, 42, 157-161.

22. Shanoja Naik, R. Pathway Distributions, Autoregressive Processes and Their Applications. PhD Thesis., Mahatma Gandhi University, Kottayam, Kerala, India, 2008.

23. Mathai, A.M.; Provost, S.B. Quadratic Forms in Random Variables: Theory and Applications; Marcel Dekker: New York, NY, USA, 2005.

24. Han, J.H. Gamma function to Beck-Cohen superstatistics. Phys. A 2013, 392, 4288-4298.

25. Sebastian, N. Limiting Approach to Generalized Gamma Bessel Model via Fractional Calculus and its Applications in Various Disciplines. 2013, arXiv:1307.7949.

26. Seema Nair, S.; Kattuveettil, A. Some remarks on the paper "On the q-type Distributions". Proc. Astrophys. Space Sci. 2010, 11-15.

27. Sebastian, N. A Generalized Multivariate Gamma Model Associated with Bessel Function. Integral Transforms and Special Functions 2011, 22, 631-645.

28. Sebastian, N. A generalized gamma model associated with Bessel function and its applications in statistical mechanics. In Proceedings of AMADE-09, Institute of Mathematics, Belarusian State Mathematics, Minsk, Belarus, 2009.

29. Anderson, W.J.; Haubold, H.J.; Mathai, A.M. Astrophysical thermonuclear functions. Astrophys. Space Sci. 1994, 214, 49-70.

30. Haubold, H.J.; John, R.W. On the evaluation of an integral connected with the thermonuclear reaction rate in the closed form. Astron. Nachr. 1978, 299, 225-232.

31. Mathai, A.M.; Haubold, H.J. Modern Problems in Nuclear and Neutrino Astrophysics; Akademie-Verlag: Berlin, Germany, 1988.

32. Mathai, A.M.; Haubold, H.J. Review of Mathematical techniques applicable in astrophysical reaction rate theory. Astrophys. Space Sci. 2002, 282, 265-280.

33. Haubold, H.J.; Mathai, A.M. The fractional kinetic equation and thermonuclear functions. Astrophys. Space Sci. 2000, 273, 53-63.

34. Haubold, H.J.; Kumar, D. Extension of thermonuclear functions through the pathway model including Maxwell-Boltzmann and Tsallis distributions. Astropart. Phys. 2008, 29, 70-76.

35. Joseph, D. P.; Haubold, H.J. Extended reaction rate integral as solutions of some general differential equations. Proc. Astrophys. Space Sci. 2009, 41-51.

36. Haubold, H.J.; Mathai, A.M. A heuristic remark on the periodic variation in the number of solar neutrinos detected on Earth. Astrophys. Space Sci. 1995, 228, 113-134.

37. Seema Nair, S. Pathway fractional integration operator. Fract. Calc. Appl. Anal. 2009, 12, 237-252.

38. Kilbas, A.A.; Kumar, D. On generalized Krätzel functions. Integral Transform. Spec. Funct. 2009, 20, 836-845.

39. Kumar, D. Type-2 P-transforms. In Proceedings of AMADE-09, Belarusian State University, Minsk, Belarus, 2009. 
40. Krätzel, E. Integral transformations of Bessel type. Generalized Functions Operational Calculus 1979, 148-155.

41. Erdélyi, A.; Magnus, W.; Oberhettinger, F.; Tricomi, F.G. Higher Transcendental Functions; McGraw-Hill: New York, NY, USA, 1953; Volume 1.

42. Kilbas, A.A.; Shlapakov, S.A. On Bessel-type integral transformation and its compositions with integral and differential operators. Dokl. Akad. Nauk Belarusi 1993, 37, 10-14.

43. Kilbas, A.A.; Saxena, R.K.; Trujillo, J.J. Krätzel function as a function of hypergeometric type. Fract. Calc. Appl. Anal. 2006, 2, 109-130.

44. Mathai, A.M. Jacobians of Matrix Transformations and Functions of Matrix Argument; World Scientific Publishing: New York, NY, USA, 1997.

45. Mathai, A.M. Random volumes under a general matrix-variate model. Linear Algebra Appl. 2007, $425,162-170$.

46. Jacob, J.; Jose, K.K.; Mathai, A.M. Some properties of real matrix-variate inverted generalized Dirichlet integral. J. Indian Acad. Math. 2004, 26, 175-189.

47. Jacob, J.; Sebastian George, S.; Mathai, A.M. Some properties of complex matrix-variate generalized Dirichlet integrals. Proc. Indian Acad. Sci. 2005, 15, 1-9.

48. Kurian, K.M.; Benny, K.; Mathai, A.M. A matrix-variate extension of inverted Dirichlet integral. Proc. Natl. Acad. Sci. (India) 2004, 74, 1-10.

49. Thomas, S.; Thannippara, A.; Mathai, A.M. On a matrix-variate generalized type-2 Dirichlet model. Adv. Appl. Stat. 2008, 8, 37-56.

50. Mathai, A.M. Fractional integrals in the matrix-variate cases and connection to statistical distributions. Integral Transforms Spec. Funct. 2009, 20, 871-882.

51. Seema Nair, S. Pathway fractional integral operator and matrix-variate functions. Integr. Transform. Spec. Funct. 2011, 22, 233-244.

52. Kober, H. On fractional integrals and derivatives. Q. J. Math. 1940, 11, 193-211.

53. Saigo, M. A remark on integral operators involving the Gauss hypergeometric functions. Math. Rep. Kyushu Univ. 1978, 11, 135-143.

(c) 2015 by the authors; licensee MDPI, Basel, Switzerland. This article is an open access article distributed under the terms and conditions of the Creative Commons Attribution license (http://creativecommons.org/licenses/by/4.0/). 